\title{
A Survey of Nonstandard Sequent Calculi
}

\begin{abstract}
The paper is a brief survey of some sequent calculi (SC) which do not follow strictly the shape of sequent calculus introduced by Gentzen. We propose the following rough classification of all SC: Systems which are based on some deviations from the ordinary notion of a sequent are called generalised; remaining ones are called ordinary. Among the latter we distinguish three types according to the proportion between the number of primitive sequents and rules. In particular, in one of these types, called Gentzen's type, we have a subtype of standard SC due to Gentzen. Hence by nonstandard ones we mean all these ordinary SC where other kinds of rules are applied than those admitted in standard Gentzen's sequent calculi. We describe briefly some of the most interesting or important nonstandard SC belonging to the three abovementioned types.
\end{abstract}

Keywords: Sequent Calculi, Natural Deduction, Proof Methods.

\section{Introduction}

The notion of sequent calculus (SC) is commonly, and rightly, connected with the name of Gerhard Gentzen. Quite automatically, we think of calculi with structural and logical rules introducing constants either to antecedent or to succedent of a sequent. However, one should be aware that nowadays this term may be applied not only to such calculi like original Gentzen's LJ, LK or their variants, but also to a variety of calculi which use sequents in a significantly different way than they are used in Gentzen's like calculi. Some of them are even older than Gentzen's LK (or LJ) - like Hertz's calculus - but most were invented after Gentzen, mainly for providing more flexible or natural systems for actual proof search than original Gentzen's calculus constructed rather for theoretical purposes. In this paper we survey some of the most important or interesting proposals. The paper is based on the 10th chapter of Indrzejczak [31].

Let us define an ordinary sequent calculus (SC) as a finite collection of (schemata) of (primitive) sequent rules of the form:

$S_{1}, \ldots, S_{n} / S_{n+1}, n \geq 0$, where $S_{i}, i \leq n+1$ denotes a schema of a sequent.

Special Issue: Gentzen's and Jaśkowski's Heritage

80 Years of Natural Deduction and Sequent Calculi

Edited by Andrzej Indrzejczak

Studia Logica (2014) 102: 1295-1322

DOI: $10.1007 / \mathrm{s} 11225-014-9567-\mathrm{y}$

C The Author(s) 2014. This article is published with open access at Springerlink.com 
Sequents on the left of / are premises of the rule, whereas $S_{n+1}$ is its conclusion. Note that $n$, i.e. the number of premises, may be 0 - in this case we will say on (primitive) axiomatic sequent; in case $n>0$ we will simply say on rules. Note that every SC have a nonempty set of primitive sequents and a nonempty set of rules ${ }^{1}$.

So far, the only restriction we put on the notion of a sequent calculus is that rules have unique conclusions. The next restriction is connected with the very notion of a sequent. We define it in a standard way as an ordered pair $\Gamma \Rightarrow \Delta$, where $\Gamma$ is the antecedent and $\Delta$ the succedent of a sequent. Hence we exclude from our considerations such calculi which operate on sequents having more arguments ${ }^{2}$ or being structures of different character ${ }^{3}$ or using more types of sequents in one system ${ }^{4}$.

One should also specify what kind of structures are denoted by arguments of a sequent. We allow sequences, multisets or sets of formulae but exclude from further considerations other kinds of structures. Hence we do consider neither display calculi invented by Belnap [6] (see Ciabattoni, Ramanayake, Wansing [10] in this issue) nor labelled sequent calculi of different sorts ${ }^{5}$. In general all these systems briefly referred to in this section, and do not taken into consideration in further sections, may be called generalised sequent calculi (GSC).

\footnotetext{
${ }^{1}$ In what follows for simplicity we will not distinguish between schemata of sequents/rules and their instances. Hence we will simply call a sequent/rule an infinite set of sequents/rules covered by a schema as well as a particular instance.

${ }^{2}$ There are popular systems defined for multi-valued logics with sequents having as many arguments as the number of truth-values of respective logic, see e.g. Rousseau [50], Carnielli [9]. The other group covers systems for modal and temporal logics, where the distinction is made inside antecedent and succedent to distinguish between formulae of different modality or temporal localization, see e.g. Blamey and Humberstone [8] or Nishimura [43]. One can also consider as a separate group systems which use one-sided sequents (see e.g. Schütte [54] or Tait [63]) but in all cases where formalised logic has standard negation, these calculi may be considered as simply notational variant of calculi with standard (i.e. two-sided) sequents. Note however that in the context of some substructural logics, like Linear Logic without Permutation rules, formalizations based on one-sided sequents tend to behave better than those based on standard sequents, e.g. Hudelmayer and Schroeder-Heister [27] showed that the former admits cut-elimination, whereas the latter does not.

${ }^{3}$ These include e.g. hypersequent calculi developed by Avron [5] (see the survey of Ciabattoni, Ramanayake, Wansing [10] in this issue) or several variants of nested sequent calculi like in: Došen [12], Kashima [34], Stouppa [57] or Poggiolesi [45].

${ }^{4}$ The approach starting with Curry [11] and developed by Indrzejczak [28] for modal and temporal logics.

${ }^{5}$ General theory of labelled systems is presented in Gabbay [19]; for a survey of approaches developed for modal logics see Indrzejczak [30].
} 
The remaining group of sequent calculi, called ordinary, may be classified according to many criteria. One can for example distinguish between systems which use intuitionistic sequents with at most one formula in the succedent of a sequent (many-one) and systems using classical sequents which admit more formulae (many-many $)^{6}$. Other solutions, e.g. Rieger's [49] system (see section 4.3.) using sequents with just one formula in both sides of a sequent are also possible. Another important criterion is the definition of a proof - is it a tree or a sequence of sequents. Yet another division may be carried according to the balance between structural and logical rules in the system (see, e.g. Poggiolesi [45]). In what follows we propose a typology based on the proportion between the number of primitive sequents and rules in the system. One can distinguish three types of ordinary SC:

1. Gentzen's type based on rules with small number (usually one) of primitive sequents;

2. Hertz's type based on primitive sequents with small number of rules;

3. Mixed type.

Usually in the first type logical constants are characterised (mainly) by rules with primitive sequents of structural character, whereas in the second type, on the contrary, rules have structural character and logical constants are characterised by primitive sequents. Gentzen's original LJ or LK and their variants applied in proof-theoretical works belong to the first type but satisfy additional important feature which we call progressivity of logical rules. It means that logical constants are characterised only by means of rules of introduction to the conclusion; usually a pair introducing a constant to an antecedent or to a succedent. All calculi of the first type with only progressive logical rules are called standard SC. This subtype of the first type is well known and their important properties like cut admissibility, subformula-property e.t.c. were discussed in detail in many places, whereas sequent calculi of different sort are relatively unknown. In what follows we will present some nonstandard SC, i.e. calculi belonging either to the first type but with nonprogressive rules, or belonging to the remaining two types.

\footnotetext{
${ }^{6}$ The names refer to Gentzen's original solution where a calculus LJ with sequents of the first sort was defined for intuitionistic logic, whereas LK with sequents of the second sort was developed for classical logic. Of course it is to some extent arbitrary since one can develop SC for intuitionistic logigic with many-many sequents (see e.g. Negri, von Plato [42]), and as we will see in the next sections, most of formalizations of classical logic use many-one sequents.
} 


\section{Nonstandard SC of Gentzen's type}

It covers many $\mathrm{SC}$ where the role of primitive sequents is reduced to minimum. Except standard SC we may find here the following (classes of) systems:

2.1 Sequent natural deduction of Gentzen [23];

2.1 Suppes' [58] and Lemmon's [39] natural deduction;

2.2 Systems of german logicians (Hermes [25], Ebbinghaus, Flum and Thomas [15]);

2.2 Systems of russian logicians (Ershow, Palyutin [16], Ławrow, Maksimowa [41]);

2.3 Andrews' system [3];

2.4 Leblanc's systems [37, 38];

2.5 Systems with rules for negated formulae [40, 56, 66];

2.6 Došen's structural SC [13].

\subsection{Sequent Natural Deduction of Gentzen and his followers}

We do not mean here the well known system of natural deduction NK (or NJ for intuitionist logic) which was presented by Gentzen in [22] but the system introduced in [23] which was applied in the proof of the consistency of Peano's arithmetic. This system is devised for classical logic but uses intuitionistic sequents with sequences of formulae in antecedents. It consists of the rules of introduction of logical constants only in the succedent; instead of rules for introduction in the antecedent it has the rules of elimination of constants in the succedent. Hence it seems as a kind of a compromise between his system NK of natural deduction and his standard sequent system LK in the sense that all inference rules are basically as in his NK, but items which are operated on in the proof are not formulae but sequents. Antecedents of the sequents do not involve any logical operations; they provide only a record of active assumptions.

In fact, such a system was implicitly present in [22] in the proof of correctness of NK. Gentzen shows there an equivalence of his NK with Hilbert's system via LK. One part of the proof shows how to transform every NK proof-tree into LK proof-tree. For this aim every inference rule of NK is rewritten with addition of all active assumptions, this way we obtain as a by-product the system which in [23] is defined explicitly. 
The only primitive sequents are of the form $\varphi \Rightarrow \varphi$, exactly as in LK. He also applies structural rules of weakening, contraction and permutation in antecedents - as in LJ. Logical rules are the following:

$$
\begin{aligned}
& (\Rightarrow \neg) \frac{\Gamma, \varphi \Rightarrow \psi \quad \Delta, \varphi \Rightarrow \neg \psi}{\Gamma, \Delta \Rightarrow \neg \varphi} \\
& (\Rightarrow \neg E) \frac{\Gamma \Rightarrow \neg \neg \varphi}{\Gamma \Rightarrow \varphi} \\
& (\Rightarrow \wedge E 1) \frac{\Gamma \Rightarrow \varphi \wedge \psi}{\Gamma \Rightarrow \varphi} \\
& (\Rightarrow \wedge E 2) \frac{\Gamma \Rightarrow \varphi \wedge \psi}{\Gamma \Rightarrow \psi} \\
& (\Rightarrow \wedge) \frac{\Gamma \Rightarrow \varphi \quad \Delta \Rightarrow \psi}{\Gamma, \Delta \Rightarrow \varphi \wedge \psi} \\
& (\Rightarrow \vee E) \frac{\Gamma, \varphi \Rightarrow \chi \quad \Delta, \psi \Rightarrow \chi \quad \Pi \Rightarrow \varphi \vee \psi}{\Gamma, \Delta, \Pi \Rightarrow \chi} \\
& (\Rightarrow \vee) \frac{\Gamma \Rightarrow \varphi}{\Gamma \Rightarrow \varphi \vee \psi} \\
& (\Rightarrow \vee) \frac{\Gamma \Rightarrow \psi}{\Gamma \Rightarrow \varphi \vee \psi} \\
& (\Rightarrow \rightarrow E) \quad \frac{\Gamma \Rightarrow \varphi \quad \Delta \Rightarrow \varphi \rightarrow \psi}{\Gamma, \Delta \Rightarrow \psi} \\
& (\Rightarrow \rightarrow) \quad \frac{\Gamma, \varphi \Rightarrow \psi}{\Gamma \Rightarrow \varphi \rightarrow \psi} \\
& (\Rightarrow \forall E) \quad \frac{\Gamma \Rightarrow \forall x \varphi}{\Gamma \Rightarrow \varphi[x / \tau]} \\
& (\Rightarrow \forall)^{1} \frac{\Gamma \Rightarrow \varphi[x / a]}{\Gamma \Rightarrow \forall x \varphi} \\
& (\Rightarrow \exists) \frac{\Gamma \Rightarrow \varphi[x / \tau]}{\Gamma \Rightarrow \exists x \varphi} \\
& (\Rightarrow \exists E)^{2} \quad \frac{\Gamma \Rightarrow \exists x \varphi \quad \varphi[x / a], \Delta \Rightarrow \psi}{\Gamma, \Delta \Rightarrow \psi}
\end{aligned}
$$

Side-conditions:

1. parameter (free variable) $a$ is not free in $\Gamma$.

2. parameter $a$ is not free in $\Gamma, \Delta$ and $\psi$.

It is easily seen that this calculus when compared with standard SC of Gentzen has standard rules for introduction in the succedent taken from LJ; two-premise form of $(\Rightarrow \neg$ ) follows from the fact that $\perp$ (or empty succedent) is not present in the language. Instead of rules of introduction in the antecedent we have elimination (in succedent) rules taken from Gentzen's Natural Deduction system NK (or NJ for intuitionistic logic). In particular, the rules $(\Rightarrow \neg),(\Rightarrow \rightarrow),(\Rightarrow \vee E)$ and $(\Rightarrow \exists E)$ correspond to proof construction rules in NK, i.e. these rules which introduce subderivations based on additional assumptions later discharged (after deduction of suitable formula). It is represented here as a subtraction of some formula (additional assumption) from the antecedent of a sequent. all other rules correspond to inference rules of NK (NJ) so the only operation on antecedents of premises is their concatenation.

It is easy to note that Cut-rule is derivable in this system by means of $(\Rightarrow \rightarrow)$ and $(\Rightarrow \rightarrow E)$ but of course it can be proved directly as na admissible rule of the system, independently of the special rules for implication. 
This Gentzen's system is based on the rigid conception of natural deduction as characterising logical constants only by means of rules operating only on succedents of sequents. The only operations admissible on antecedents have - as we noted above - structural character. It seems that the exception to this requirement is $(\Rightarrow \exists E)$, but it is an analogon of $(\Rightarrow \vee E)$ which is natural if we understand $\exists$ as a disjunction in finite domain.

This Sequent Natural Deduction system of Gentzen is not very practical in its original form. The drawbacks are twofold: proof is defined as a tree of sequents which is not very practical; all assumptions must be rewritten in each inference step. But the latter property allows to resign from defining a proof as a tree of sequents and use linear proofs (i.e. sequences of sequents) which are much easier to deal with from the standpoint of actual proof search. Moreover, in contrast to Natural Deduction systems in Jaśkowski style $^{7}$, it does not have to use any bookkeeping techniques, like lines or boxes, for showing the dependancy of formulae in the proof from their assumptions. Also the process of rewriting of all assumptions in each inference step may be significantly simplified since the only operations on them have structural character. Instead of rewritting formulae we may rewrite the numbers of lines where respective assumptions were introduced. These solution was first introduced in Feys' and Ladriere [17] translation of [22] into french. It was made popular due to Suppes' [58] and its later simplifications in Lemmon [39], Forbes [18] and many other textbooks. Usually in all these systems authors simplify matters by introducing sets instead of sequences of formulae/numbers in antecedents. It makes structural rules of permutation and contraction dispensable ${ }^{8}$.

Clearly, in order to simplify Gentzen's system in this way we must also change Gentzen's $(\Rightarrow \exists E$ ). Suppes proposed a rule of the form:

$$
\Gamma \Rightarrow \exists x \varphi / \Gamma \Rightarrow \varphi\left[x / a_{y_{1}, \ldots, y_{n}}\right]
$$

where we have a direct elimination of $\exists$ but it needs a skolemization since $a$ is a new parameter indexed by all free variables occuring in $\varphi$. Moreover, such a rule requires additional complications in the formulation of correct rule for introduction of $\forall$. Much better solution was provided by Lemmon, who used a rule:

$$
\Gamma \Rightarrow \exists x \varphi ; \Delta, \varphi[x / a] \Rightarrow \psi / \Gamma, \Delta \Rightarrow \psi,
$$

where $a$ is a new parameter with respect to $\Gamma, \Delta, \varphi, \psi$.

\footnotetext{
${ }^{7}$ In angloamerican tradition usually called Fitch-style Natural Deduction.

${ }^{8}$ Although one should observe that in some cases, e.g. in modal logics, it may be better to keep sequences - see Garson [20].
} 
We finish this subsection with the example of a proof in Suppes' system:

$\begin{array}{ccll}1 & \{1\} & p \rightarrow q & \text { ass. } \\ 2 & \{2\} & q \rightarrow r & \text { ass. } \\ 3 & \{3\} & p & \text { ass. } \\ 4 & \{1,3\} & q & 1,3,(\rightarrow E) \\ 5 & \{1,2,3\} & r & 2,4,(\rightarrow E) \\ 6 & \{1,2\} & p \rightarrow r & 5,(\rightarrow I) \\ 7 & \{1\} & (q \rightarrow r) \rightarrow(p \rightarrow r) & 6,(\rightarrow I) \\ 8 & \varnothing & (p \rightarrow q) \rightarrow((q \rightarrow r) \rightarrow(p \rightarrow r)) & 7,(\rightarrow I)\end{array}$

One can see that the final form of this calculus is apparently Natural Deduction performed directly on formulae, not on sequents, but it should be treated as a transformation of original Sequent Natural Deduction of Gentzen. $^{9}$

\subsection{Extended Sequent Natural Deduction of Hermes and his fol- lowers}

As we have seen the rigid Gentzen's int-elim scheme for the rules operating only on succedents of the sequents leads to simplifications by reduction of antecedents to sets of numbers. On the other hand, in order to obtain more flexible tool for actual proof search one can admit the possibility of making logical operations also in antecedents. Such a solution was examined by some german and russian logicians. It seems that the first system of this sort was provided by Hermes [25]. He also uses intuitionistic sequents with sequences of formulae in antecedents in his formalization of classical logic with identity. As primitive sequents Hermes uses only: $\varphi \Rightarrow \varphi$ and $\Rightarrow \tau=\tau$. Logical rules are the following:

$$
\begin{array}{lr}
(\neg E \Rightarrow) \frac{\Gamma, \varphi \Rightarrow \psi \quad \Delta, \neg \varphi \Rightarrow \psi}{\Gamma, \Delta \Rightarrow \psi} & (\Rightarrow \neg E) \frac{\Gamma \Rightarrow \varphi \quad \Delta \Rightarrow \neg \varphi}{\Gamma, \Delta \Rightarrow \psi} \\
(\Rightarrow \wedge E 1) \frac{\Gamma \Rightarrow \varphi \wedge \psi}{\Gamma \Rightarrow \varphi} & (\Rightarrow \wedge E 2) \frac{\Gamma \Rightarrow \varphi \wedge \psi}{\Gamma \Rightarrow \psi} \\
(\Rightarrow \wedge) \frac{\Gamma \Rightarrow \varphi \Delta \Rightarrow \psi}{\Gamma, \Delta \Rightarrow \varphi \wedge \psi} & (\vee \Rightarrow) \frac{\Gamma, \varphi \Rightarrow \chi \quad \Delta, \psi \Rightarrow \chi}{\Gamma, \Delta, \varphi \vee \psi \Rightarrow \chi}
\end{array}
$$

\footnotetext{
${ }^{9}$ One can treat also Natural Deduction systems for relevant logics of Anderson and Belnap [2] as belonging to this class due to the fact that all formulae have added a set of (numbers) of active assumptions. However in this case the additional structure of subproofs, characteristic for standard Natural Deduction on formulae, is also saved due to restrictions needed for reiteration rule.
} 


$$
\begin{aligned}
& (\Rightarrow \vee) \frac{\Gamma \Rightarrow \varphi}{\Gamma \Rightarrow \varphi \vee \psi} \\
& (\Rightarrow \vee) \quad \frac{\Gamma \Rightarrow \psi}{\Gamma \Rightarrow \varphi \vee \psi} \\
& (\rightarrow \Rightarrow) \frac{\Gamma, \neg \varphi \Rightarrow \chi \quad \Delta, \psi \Rightarrow \chi}{\Gamma, \Delta, \varphi \rightarrow \psi \Rightarrow \chi} \\
& (\Rightarrow \rightarrow) \quad \frac{\Gamma, \varphi \Rightarrow \psi}{\Gamma \Rightarrow \varphi \rightarrow \psi} \\
& (\Rightarrow \forall E) \frac{\Gamma \Rightarrow \forall x \varphi}{\Gamma \Rightarrow \varphi} \\
& (\Rightarrow \forall)^{1} \quad \frac{\Gamma \Rightarrow \varphi}{\Gamma \Rightarrow \forall x \varphi} \\
& (\Rightarrow \exists) \frac{\Gamma \Rightarrow \varphi}{\Gamma \Rightarrow \exists x \varphi} \\
& (\exists \Rightarrow)^{2} \frac{\Gamma, \varphi \Rightarrow \psi}{\Gamma, \exists x \varphi \Rightarrow \psi} \\
& (\Rightarrow=) \frac{\Gamma \Rightarrow \varphi}{\Gamma, x=\tau \Rightarrow \varphi[x / \tau]} \\
& (\text { Subst })^{3} \frac{\Gamma \Rightarrow \varphi}{\Gamma^{\prime} \Rightarrow \varphi^{\prime}}
\end{aligned}
$$

Side-conditions:

1. $x$ not free in $\Gamma$.

2. $x$ not free in $\Gamma$ and $\psi$.

3. $\Gamma^{\prime}, \varphi^{\prime}$ obtained by substitution from $\Gamma, \varphi$.

Although proofs in Hermes' system are defined as linear sequences of sequents we use tree representation as theoretically superior in simple cases:

We can easily notice that $\vee$ and $\exists$ is characterised exactly as in Gentzen's LJ, whereas $\wedge$ and $\forall$ like in Gentzen's sequent natural deduction, with the only difference that in all rules for quantifiers no substitution is allowed since this operation is defined by a separate rule. $\rightarrow$ is characterised almost as in Gentzen's LJ, but $(\rightarrow \Rightarrow)$ displays $\neg$ in one of the premise. $\neg$ is characterised in nonstandard way; $(\neg E \Rightarrow)$ is a variant of proof by cases, and $(\Rightarrow \neg E)$ is a variant of Duns Scotus rule.

Very unusual feature of Hermes' system is the lack of structural rules although sequents use sequences of fromulae in antecedents. It appears that all rules are derivable. Drivability of permutation follows from the way the substitutivity is defined by Hermes and from the application of (Subst). Derivability of weakening is shown below:

$$
\frac{\Gamma \Rightarrow \varphi \quad \psi \Rightarrow \psi}{\frac{\Gamma, \psi \Rightarrow \varphi \wedge \psi}{\Gamma, \psi \Rightarrow \varphi}(\Rightarrow \wedge E)}(\Rightarrow \wedge)
$$

Although Modus Ponens is not a primitive rule of a system cut-rule is simply derivable:

$$
(\neg E \Rightarrow) \frac{\Delta, \varphi \Rightarrow \psi \quad \frac{\Gamma \Rightarrow \varphi \quad \neg \varphi \Rightarrow \neg \varphi}{\Gamma, \neg \varphi \Rightarrow \psi}}{\Gamma, \Delta \Rightarrow \psi}(\Rightarrow \neg E)
$$


One can observe that both rules for $\neg$ are strong enough to simulate cut and to allow also the proof of Modus Ponens as derivable rule. Hermes in fact shows derivability of many rules in order to increase the flexibility of proof search in his system. For example, he introduces as derivable the following rules for $\wedge$ :

$$
(\wedge \Rightarrow) \frac{\Gamma, \varphi, \psi \Rightarrow \chi}{\Gamma, \varphi \wedge \psi \Rightarrow \chi} \quad(\wedge E \Rightarrow) \frac{\Gamma, \varphi \wedge \psi \Rightarrow \chi}{\Gamma, \varphi, \psi \Rightarrow \chi}
$$

Very similar system for classical logic with $\neg, \vee, \exists$ and linear proofs contains [15]. The following differences should be noticed:

1. All two-premise rules have the same antecedents, i.e. context-sharing. ${ }^{10}$

2. The preceding property makes necessary the addition of weakening as a primitive rule since it is not longer derivable (see the proof in Hermes' system where the rule for $\wedge$ was context-free), but it is necessary for unifying antecedents of sequents which we want to use as premises for application of two-premise rules. In Ebbinghaus, Flum and Thomas' system we have one structural rule which allows contraction, permutation and weakening on the antecedent of any sequent. The only primitive sequent has the form $\Gamma \Rightarrow \varphi$, where $\varphi \in \Gamma$.

3. Rules for negation are similar; $(\neg E \Rightarrow$ ) (called the rule of prof by cases) is the same as in Hermes (but with unified antecedents), $(\Rightarrow \neg E)$ (called the rule of contradiction) has a slightly different form:

$$
(\neg E \Rightarrow) \frac{\Gamma, \varphi \Rightarrow \psi \quad \Gamma, \neg \varphi \Rightarrow \psi}{\Gamma \Rightarrow \psi} \quad(\Rightarrow \neg E) \frac{\Gamma, \neg \psi \Rightarrow \varphi \quad \Gamma, \neg \psi \Rightarrow \neg \varphi}{\Gamma \Rightarrow \psi}
$$

Hermes' rule $(\Rightarrow \neg E)$ (with unified antecedents) is derivable as the second rule of contradiction, similarly as Cut (called the chain rule). In the first proof it suffices to add $\neg \psi$ to antecedents of both premises by weakening, and then apply $(\Rightarrow \neg E)$; the proof of derivability of Cut goes as in Hermes.

4. The remaining rules (for $\vee, \exists$ and $=$ ) are like in Hermes' system but without separate rule of substitution; the effect of this operation is involved in suitable rules for quantifiers - as in Gentzen's system.

Quite similar systems of sequent natural deduction are also popular form of textbook presentation of logic in Russia, e.g. Ershow, Palyutin [16] or Ławrow, Maksimowa [41]. In the latter a propositional part of classical logic is characterised almost identical as in Gentzen's system from subsection 2.1,

\footnotetext{
${ }^{10}$ Althouhg we do not consider in this paper systems for logics weaker than intuitionistic one could also use in this context the name additive rules, in contrast to multiplicative, i.e. context free rules, preferred by Hermes.
} 
in the former system with two-premise rules having the same antecedents. In both systems $\neg$ is characterised by different pair of rules:

$$
(\neg E \Rightarrow) \frac{\Gamma, \neg \varphi \Rightarrow}{\Gamma \Rightarrow \varphi} \quad(\Rightarrow \neg E) \frac{\Gamma \Rightarrow \varphi \quad \Gamma \Rightarrow \neg \varphi}{\Gamma \Rightarrow}
$$

The extension to first-order logic is made differently in both systems. Ershow, Palyutin [16] apply standard Gentzen's rules from LJ; in fact they introduce also a lot of derivable rules for propositional part which introduce or eliminate logical constants in antecedents. Lawrow, Maksimowa [41] characterize $\exists$ as in LJ, but $\forall$ like in sequent natural deduction, i.e. by introduction and elimination in succedent only. In both approaches tree format for proofs is saved.

\subsection{Andrews' system}

It is a system for classical logic of quite different character from all discussed above. Andrews uses intuitionistic sequents with sequences in antecedents, proofs are linear and all many-premise rules have the same antecedents. The set of primitive rules comprises:

1. In the class of structural rules we have one rule which allows for making permutation, contraction and weakening in the antecedent. It is only one primitive sequent of the form $\Gamma \Rightarrow \varphi$, where $\varphi \in \Gamma$, as in Ebbinghaus, Flum's system.

2. Connectives are characterised by means of three logical rules which correspond to proof construction rules in ordinary natural deduction system. These are: $(\Rightarrow \rightarrow),(\Rightarrow \vee E)$ (with the same antecedents) and for $\neg$ :

$(\neg E \Rightarrow) \frac{\Gamma, \neg \varphi \Rightarrow \perp}{\Gamma \Rightarrow \varphi}$

Instead of separate rules which are counterparts of inference rules from natural deduction, Andrews uses one rule:

(Taut) $\frac{\Gamma \Rightarrow \varphi_{1}, \ldots, \Gamma \Rightarrow \varphi_{n}}{\Gamma \Rightarrow \psi}$

where $\varphi_{1} \wedge \ldots \wedge \varphi_{n} \rightarrow \psi$ is any classical tautology ${ }^{11}$.

3. The characterization of quantifiers is based on rules of elimination similar to those in Gentzen system (see section 2.1.), but with $(\Rightarrow \exists E$ )

\footnotetext{
${ }^{11}$ Similar solutions are used by many authors of popular natural deduction systems, e.g. Quine [48].
} 
having the same antecedents in both premises. The rules of introduction have a generalised form:

$$
(\Rightarrow \forall G) \frac{\Gamma \Rightarrow \psi \vee \varphi[x / a] \vee \chi}{\Gamma \Rightarrow \psi \vee \forall x \varphi \vee \chi} \quad(\Rightarrow \exists G) \frac{\Gamma \Rightarrow \psi \vee \varphi[x / \tau] \vee \chi}{\Gamma \Rightarrow \psi \vee \exists x \vee \chi}
$$

Clearly, $(\Rightarrow \forall G)$ must satisfy the condition of $a$ being new; also in both rules we admit that $\psi$ or $\chi$ is not present. Additionaly, in the set of primitive rules there is a rule covering the effect of four De Morgan's rules for quantifiers, and a rule of change of any sequent for its alphabetic variant.

It is easily seen that the system is redundant. Int-elim schema for logical rules is kept only for quantifiers; in propositional part only these rules are present which correspond to proof construction rules in Natural Deduction.

\subsection{Leblanc's system}

Leblanc $[37,38]$ introduced a variant of Gentzen's LK but on intuitionistic sequents, which may be seen as a kind of sequent natural deduction but with generalised rules of elimination. The differences with Gentzen's system are the following:

1. All many-premise rules have the same antecedents.

2. In $(\Rightarrow \forall)$ and $(\Rightarrow \exists E)$ there is no substitution of a new parameter $a$ for $x$, but $x$ must satisfy the condition of being absent in $\Gamma$ (and $\psi$ in $(\Rightarrow \exists E))$ as in Hermes' system. Both rules have the following form:

$$
(\Rightarrow \forall) \frac{\Gamma \Rightarrow \varphi}{\Gamma \Rightarrow \forall x \varphi} \quad(\Rightarrow \exists E) \frac{\Gamma \Rightarrow \exists x \varphi \quad \Gamma, \varphi \Rightarrow \psi}{\Gamma \Rightarrow \psi}
$$

3. Rules for $\neg$ and $\vee$ are like in Gentzen's system, but with $(\Rightarrow \neg)$ and $(\Rightarrow \vee E)$ with the same antecedents). In the remaining cases we have the following rules of elimination:

$$
\begin{aligned}
& (\Rightarrow \forall E) \frac{\Gamma \Rightarrow \forall x \varphi \quad \Gamma, \varphi[x / y] \Rightarrow \psi}{\Gamma \Rightarrow \psi} \quad(\Rightarrow \wedge E) \frac{\Gamma \Rightarrow \varphi \wedge \psi \quad \Gamma, \varphi, \psi \Rightarrow \chi}{\Gamma \Rightarrow \chi} \\
& (\Rightarrow \rightarrow E) \frac{\Gamma \Rightarrow \varphi \rightarrow \psi \quad \Gamma \Rightarrow(\varphi \rightarrow \chi) \rightarrow \varphi}{\Gamma \Rightarrow \psi}
\end{aligned}
$$

Specific form of rules of elimination for $\rightarrow, \wedge \mathrm{i} \forall$ is based on the schema of elimination rules for $\vee$ and $\exists$. Introduction of such rules allows for proving an interesting property which is a weaker version of subformula property. For any propositional sequent $S$ which is clasically provable we can find a proof in which every constant occuring in any sequent of this proof occurs also in $S$. This result does not hold for first-order system. Let us note that 
the characterization of $\neg$ by means of a rule of elimination of double negation is not sufficient for obtaining the characterization of classical implication by means of Modus Ponens (like in Gentzen's system). In Leblanc's system the replacement of his $(\Rightarrow \rightarrow E)$ by Modus Ponens gives intuitionistic implication. Of course if we want to obtain a full system for intuitionistic logic we must also weaken elimination rules for $\neg$; instead of double elimination rule we must use:

$(\Rightarrow \neg E) \frac{\Gamma \Rightarrow \varphi \quad \Gamma \Rightarrow \neg \varphi}{\Gamma \Rightarrow \psi}$

It is worth mentioning that interesting application of such generalised elimination rules but in standard natural deduction is provided by Negri and von Plato [42]. Let us note that in this context every elimination rule is a proof construction rule since we do not break every compound formula directly but by means of subproofs initiated with respective subformulae. Von Plato [44] has shown that such a solution allows much simpler proof of normalization theorem for natural deduction system.

\subsection{Systems with rules for negated formulae}

Smullyan [56] introduced a variant of SC called symmetric which was particularly useful for proving interpolation theorems. It is similar to Gentzen's standard SC with respect to the shape of rules; there are only introduction rules which are divided into antecedent- and succedent-operating. But there are no rules which involve a transfer of any formula from one side of a sequent into the other. Consequently negation has a special treatment and Gentzen's rules for implication are modified. There are no standard introduction rules for negation, instead we have a pair of rules for introduction of negated complex formulae of any kind (hence also two rules for introduction of double negation). Except usual axioms with contexts there are also sequents of the form: $\Gamma, \varphi, \neg \varphi \Rightarrow \Delta$ and $\Gamma \Rightarrow \Delta, \varphi, \neg \varphi$. Rules for $\wedge$ and $\vee$ are standard (context sharing in case of two-premises, and with both components of introduced formula in premises). For $\rightarrow$ we have the following (symmetric) rules:

$$
(\Rightarrow \rightarrow) \frac{\Gamma \Rightarrow \Delta, \neg \varphi, \psi}{\Gamma \Rightarrow \Delta, \varphi \rightarrow \psi} \quad(\rightarrow \Rightarrow) \frac{\Gamma, \neg \varphi \Rightarrow \Delta \quad \Gamma, \psi \Rightarrow \Delta}{\Gamma, \varphi \rightarrow \psi \Rightarrow \Delta}
$$

For negated formulae we have the following rules:

$$
(\Rightarrow \neg \wedge) \quad \frac{\Gamma \Rightarrow \Delta, \neg \varphi, \neg \psi}{\Gamma \Rightarrow \Delta, \neg(\varphi \wedge \psi)} \quad(\neg \wedge \Rightarrow) \quad \frac{\Gamma, \neg \varphi \Rightarrow \Delta \quad \Gamma, \neg \psi \Rightarrow \Delta}{\Gamma, \neg(\varphi \wedge \psi) \Rightarrow \Delta}
$$




$$
\begin{aligned}
& (\neg \vee \Rightarrow) \frac{\Gamma, \neg \varphi, \neg \psi \Rightarrow \Delta}{\Gamma, \neg(\varphi \vee \psi) \Rightarrow \Delta} \quad(\Rightarrow \neg \vee) \frac{\Gamma \Rightarrow \Delta, \neg \varphi \quad \Gamma \Rightarrow \Delta, \neg \psi}{\Gamma \Rightarrow \Delta, \neg(\varphi \vee \psi)} \\
& (\neg \rightarrow \Rightarrow) \frac{\Gamma, \varphi, \neg \psi \Rightarrow \Delta}{\Gamma, \neg(\varphi \rightarrow \psi) \Rightarrow \Delta} \quad(\Rightarrow \neg \rightarrow) \frac{\Gamma \Rightarrow \Delta, \varphi \quad \Gamma \Rightarrow \Delta, \neg \psi}{\Gamma \Rightarrow \Delta, \neg(\varphi \rightarrow \psi)} \\
& (\neg \neg \Rightarrow) \begin{array}{c}
\Gamma, \varphi \Rightarrow \Delta \\
\Gamma, \neg \neg \varphi \Rightarrow \Delta
\end{array} \quad(\Rightarrow \neg \neg) \quad \begin{array}{l}
\Gamma \Rightarrow \Delta, \varphi \\
\Gamma \Rightarrow \Delta, \neg \neg \varphi
\end{array}
\end{aligned}
$$

Formalization of first-order logic is obtained by addition of eight rules. $(\Rightarrow \forall)$ and $(\exists \Rightarrow)$ are standard Gentzen's rules; the remaining ones are:

$$
\begin{aligned}
& (\forall \Rightarrow) \frac{\Gamma, \forall x \varphi, \varphi[x / \tau] \Rightarrow \Delta}{\Gamma, \forall x \varphi \Rightarrow \Delta} \quad(\Rightarrow \exists) \frac{\Gamma \Rightarrow \Delta, \exists x \varphi, \varphi[x / \tau]}{\Gamma \Rightarrow \Delta, \exists x \varphi} \\
& (\neg \forall \Rightarrow) \begin{array}{ll}
\Gamma, \exists x \neg \varphi \Rightarrow \Delta \\
\Gamma, \neg \forall x \varphi \Rightarrow \Delta
\end{array} \quad(\Rightarrow \neg \forall) \quad \begin{array}{l}
\Gamma \Rightarrow \Delta, \exists x \neg \varphi \\
\Gamma \Rightarrow \Delta, \neg \forall x \varphi
\end{array} \\
& (\neg \exists \Rightarrow) \frac{\Gamma, \forall x \neg \varphi \Rightarrow \Delta}{\Gamma, \neg \exists x \varphi \Rightarrow \Delta} \quad(\Rightarrow \neg \exists) \frac{\Gamma \Rightarrow \Delta, \forall x \neg \varphi}{\Gamma \Rightarrow \Delta, \neg \exists x \varphi}
\end{aligned}
$$

This system has no primitive structural rules (including cut), all logical rules are invertible and it satisfies strong generalised subformula-property. It is generalised in the sense that all rules are closed on subformulae and their single negations; it is strong in the sense that all formulae in the antecedent are built from formulae occurring in the antecedent only, and similarly for formulae in the succedent.

Wiśniewski [66] (and [40] with Leszczyńska-Jasion and Urbański) extracted a similar sequent system for classical logic from the deduction system of generalised character provided for the system of the logic of questions. In contrast to Smullyan's system it is not symmetric because it is defined on intuitionistic sequents but also no special rules for negation are offered in order to obtain classical logic. In fact sequents are composed of finite sequences of formulae in antecedents but we will use sets as in Smullyan's solution. There are only logical introduction rules which add complex formulae or their negations to antecedent or succedent of a sequent. The system $G^{*}$ for classical propositional logic consists of axioms of the form $\Gamma, \varphi \Rightarrow \varphi$ or $\Gamma, \varphi, \neg \varphi \Rightarrow \psi$ and standard Gentzen (intuitionistic) rules $(\Rightarrow \wedge),(\wedge \Rightarrow),(\vee \Rightarrow)$ and $(\Rightarrow \rightarrow)$ with the same contexts in both premises. For $(\Rightarrow \vee)$ and $(\rightarrow \Rightarrow)$ we have the following:

$$
(\Rightarrow \vee) \frac{\Gamma, \neg \varphi \Rightarrow \psi}{\Gamma \Rightarrow \varphi \vee \psi} \quad(\rightarrow \Rightarrow) \frac{\Gamma, \neg \varphi \Rightarrow \chi \quad \Gamma, \psi \Rightarrow \chi}{\Gamma, \varphi \rightarrow \psi \Rightarrow \chi}
$$


For negated formulae we have the following rules:

$$
\begin{aligned}
& (\Rightarrow \neg \wedge) \frac{\Gamma, \varphi \Rightarrow \neg \psi}{\Gamma \Rightarrow \neg(\varphi \wedge \psi)} \quad(\neg \wedge \Rightarrow) \frac{\Gamma, \neg \varphi \Rightarrow \chi \quad \Gamma, \neg \psi \Rightarrow \chi}{\Gamma, \neg(\varphi \wedge \psi) \Rightarrow \chi} \\
& (\neg \vee \Rightarrow) \frac{\Gamma, \neg \varphi, \neg \psi \Rightarrow \chi}{\Gamma, \neg(\varphi \vee \psi) \Rightarrow \chi} \quad(\Rightarrow \neg \vee) \frac{\Gamma \Rightarrow \neg \varphi \quad \Gamma \Rightarrow \neg \psi}{\Gamma \Rightarrow \neg(\varphi \vee \psi)} \\
& (\neg \rightarrow \Rightarrow) \frac{\Gamma, \varphi, \neg \psi \Rightarrow \chi}{\Gamma, \neg(\varphi \rightarrow \psi) \Rightarrow \chi} \quad(\Rightarrow \neg \rightarrow) \frac{\Gamma \Rightarrow \varphi \quad \Gamma \Rightarrow \neg \psi}{\Gamma \Rightarrow \neg(\varphi \rightarrow \psi)} \\
& (\neg \neg \Rightarrow) \frac{\Gamma, \varphi \Rightarrow \chi}{\Gamma, \neg \neg \varphi \Rightarrow \chi} \quad(\Rightarrow \neg \neg) \frac{\Gamma \Rightarrow \varphi}{\Gamma \Rightarrow \neg \neg \varphi}
\end{aligned}
$$

Strengthening to first-order logic is obtained by addition of eleven rules. $(\Rightarrow \forall)$ and $(\exists \Rightarrow)$ are standard Gentzen's introduction rules. The remaining two are:

$$
(\forall \Rightarrow) \frac{\Gamma, \forall x \varphi, \varphi[x / \tau] \Rightarrow \chi}{\Gamma, \forall x \varphi \Rightarrow \chi} \quad(\Rightarrow \exists) \frac{\Gamma, \forall x \neg \varphi \Rightarrow \varphi[x / \tau]}{\Gamma \Rightarrow \exists x \varphi}
$$

Additionaly there are four DeMorgan rules, as in Smullyan's system, and four rules for addition of vacuous quantifier to a formula. The system is well suited for automatic proof-search since it is cut-free and satisfies generalised subformula-property. Moreover, it shows how to obtain a formulation of classical logic on intuitionistic sequents without introducing any special rules. One can conclude with the remark that the idea of using special rules for introduction of negated formulae appeared useful in formalization of some nonclassical logics with special kinds of negation, but it is beyond the scope of this paper.

\subsection{Structural system of Došen}

Došen [13] proposed a structural version of LK in the language without negation but with $\perp$. In this system the set of structural rules is not eliminable. Every constant is characterised by means of only one, but double-line (i.e. invertible) rule:

$$
\begin{aligned}
& (\rightarrow) \frac{\varphi, \Gamma \Rightarrow \Delta, \psi}{\Gamma \Rightarrow \Delta, \varphi \rightarrow \psi} \quad(\wedge) \frac{\Gamma \Rightarrow \Delta, \varphi \quad \Gamma \Rightarrow \Delta, \psi}{\Gamma \Rightarrow \Delta, \varphi \wedge \psi} \\
& (\vee) \frac{\varphi, \Gamma \Rightarrow \Delta \quad \psi, \Gamma \Rightarrow \Delta}{\varphi \vee \psi, \Gamma \Rightarrow \Delta}
\end{aligned}
$$

In each case in addition to the rule of introduction we have also a rule of elimination if we read the rule upside down. Every rule is then a counterpart 
of suitable equivalence characterising respective constant on the ground of theory of Scott's consequence relations (see [55] or [4]). The remaining rules for introduction are easily derivable by means of cut.

Došen's system serves as an exemplification of his theory of criteria of logicality. The starting point of analysis of logical constants is the conviction that logic is the science of formal proofs. Formal proof is a proof of structural character, i.e. where only structural rules were applied ${ }^{12}$. An expression is logical iff it is analysable in purely structural terms. His rules provide such an analysis in the sense that on the one side we have only structural sequents, i.e. with no constant displayed. According to Došen, in order to claim that an expression is a logical constant it is necessary to find such a doublevalid rule which after adding to structural rules allows for obtaining a full characterisation of this constant. Note that many expressions commonly claimed to be logical do not satisfy this criterion of logicality; for example modal constants. But one can find more stisfying solutions on the ground of generalised formalisations briefly mentioned in the introduction (see e.g. Wansing [64, 65] or Poggiolesi [45].)

It is worth mentioning that similar idea appeared earlier in Popper (e.g. $[46,47])$, but it was not so explicitly articulated and connected with rather unsatisfying conception of proof-theoretical semantics. Popper's project was criticised by Kleene, Curry and many others because of its inconistency. But, as was convincingly shown by Schroeder-Heister [51] (see also [53], Popper's works contain an interesting proposal for criteria of being a logical constant. Popper tried to characterize constants by means of inferential definitions which yield double-valid rules characterising constants of the form:
$(\rightarrow) \frac{\varphi, \chi \Rightarrow \psi}{\overline{\chi \Rightarrow \varphi \rightarrow \psi}}$
$(\wedge) \frac{\varphi, \psi \Rightarrow \chi}{\underline{\varphi \wedge \psi \Rightarrow \chi}}$
( $) \frac{\varphi \Rightarrow \chi \quad \psi \Rightarrow \chi}{\varphi \vee \psi \Rightarrow \chi}$
(ᄀ) $\frac{\varphi, \psi \Rightarrow \chi}{\neg \chi, \psi \Rightarrow \neg \varphi}$

\section{Structural type of Hertz's}

Systems of the first type represent a rule-based solution, where one or two types of primitive sequents are sufficient to start a proof. Quite a contrary solution was proposed by Hertz [26]. In this approach rules have purely structural character and all logical content is contained in primitive sequents.

\footnotetext{
${ }^{12}$ It is in a sense a development of Hertz's program which will be discussed in the next section.
} 
That's why, this type of SC may be called purely structural. ${ }^{13}$

Hertz introduced the notion of a sequent (of intuitionistic type), sequent rule, tree representation of proofs and started the program developed later by Gentzen. ${ }^{14}$ But it should be noted that Hertz did not present any specific system for concrete logic. His approach was abstract; he defined rather a schema of the system in which the only rules have purely structural character. Except rules of contraction, permutation and weakening in antecedent, Hertz used also the following rule of syllogism which is a kind of a cut rule:

$$
\frac{\Gamma_{1} \Rightarrow \varphi_{1} \ldots \Gamma_{n} \Rightarrow \varphi_{n}, \quad \varphi_{1}, \ldots, \varphi_{n}, \Delta \Rightarrow \psi}{\Gamma_{1}, \ldots, \Gamma_{n}, \Delta \Rightarrow \psi}
$$

Gentzen in his earlier work [21] developed to some extent theory of Hertz. In particular, he presented purely structural criteria which must be satisfied by any SC of this type with independent set of axiomatic sequents. Also he replaced Hertz's rule of syllogism with Cut which is its particular instance with $n=1$.

The approach of Hertz to characterization of concrete logics did not find application, except one significant example which we describe below. But his approach is commonly used as a way of defining theories on the ground of rule-based (i.e. of the first type, usually standard) formalization of logic.

\subsection{Suszko's system}

It seems that the only concrete realization of Hertz's idea with respect to specific logics was due to Suszko although he did not mention Hertz but rather refers to some ideas of Ajdukiewicz [1]. ${ }^{15}$ In 40ties Suszko proposed in his Ph.D. thesis [61] (see also [60] and [62]) the original SC operating on intuitionistic sequents with sequences in the antecedents. In order to understand properly his motivations one should note that in his system sequents are seen as expressing inference rules and neither antecedent (a list of premises) nor succedent (a conclusion) may be empty. His attempts resulted from dissatisfaction with the earlier systems of Gentzen and Jaśkowski. The problem he posed was called by him the problem of logic without axioms and may be expressed as follows: Is it possible to build a calculus for classical logic in which all the logical axioms are dispensed with? Instead of logical axioms only proper inference rules must be assumed as primitive. Theses of

\footnotetext{
${ }^{13}$ But it should be distinguished from the sense of structurality involved in Došen's proposal.

${ }^{14}$ One can find a detailed overview of Hertz' system in Schroeder-Heister [52].

${ }^{15}$ This subsection is based on Indrzejczak [29].
} 
logic (including axioms) may be deduced by means of these rules from arbitrary formulae. It is a way of expressing the idea that analytical sentences (= logical theses) are implied by any sentences.

In Suszko's opinion all nonaxiomatic systems proposed so far comprises some rules which are not proper. But what is a proper inference rule? According to Suszko's view, a rule is proper if it is represented by a schema, where all premises and conclusion are schemata of contingent formulae only. He claims that, e.g. proof construction rules comprising Deduction Theorem are not proper in this sense because a conclusion of its application is an implicational thesis. The same applies to other rules of this sort like Indirect Proof or Proof by Cases. Suszko is referring here to Ajdukiewicz's view from [1] and treats such rules as examples of axiomatic postulates. It seems that the objection against such rules is exagerated since schemata of them do not exhibit schemata of theses in conclusion, moreover not all applications of them lead to theses (e.g. if not all assumptions are discharged). Anyway, Suszko rejected such kind of rules from his system, hence it is certainly not a system of natural deduction in ordinary sense.

In [60] Suszko presented only adequate formalization of classical propositional logic in the language with implication and negation, comprising the following seven sequents:

$$
\begin{aligned}
& \text { 1.1.1. } \\
& \text { 1.1.2. } \quad(\varphi \rightarrow \psi) \rightarrow \chi \Rightarrow \psi \rightarrow \chi \\
& \text { 1.1.3. } \varphi \rightarrow(\psi \rightarrow \chi) \Rightarrow(\varphi \rightarrow \psi) \rightarrow(\varphi \rightarrow \chi) \\
& \text { 1.1.4. } \varphi \rightarrow \psi \quad \Rightarrow \quad(\chi \rightarrow \varphi) \rightarrow((\psi \rightarrow \gamma) \rightarrow(\chi \rightarrow \psi)) \\
& \text { 1.1.5. } \varphi, \varphi \rightarrow \psi \Rightarrow \psi \\
& \text { 1.1.6. } \quad(\varphi \rightarrow \psi) \rightarrow \chi \quad \Rightarrow \quad \neg \varphi \rightarrow \chi \\
& \text { 1.1.7. } \varphi \rightarrow \psi, \neg \varphi \rightarrow \psi \Rightarrow \psi
\end{aligned}
$$

In [61] the rest of the rules are supplied. For other propositional constants we have the following sequents:

$$
\varphi \rightarrow \psi \Rightarrow \varphi \wedge \chi \rightarrow \psi
$$

1.2.3. $\varphi \rightarrow(\psi \rightarrow \chi), \gamma \rightarrow(\psi \rightarrow \delta) \quad \Rightarrow \quad \varphi \rightarrow(\gamma \rightarrow(\psi \rightarrow \chi \wedge \delta))$

$$
\begin{aligned}
& (\varphi \rightarrow \psi) \rightarrow \chi \quad \Rightarrow \quad(\varphi \leftrightarrow \psi) \rightarrow \chi \\
& (\varphi \rightarrow \psi) \rightarrow \chi \Rightarrow(\psi \leftrightarrow \varphi) \rightarrow \chi
\end{aligned}
$$

$$
\varphi \rightarrow(\psi \rightarrow \chi), \gamma \rightarrow(\chi \rightarrow \psi) \Rightarrow \varphi \rightarrow(\gamma \rightarrow(\psi \leftrightarrow \chi))
$$

1.4.3. $\varphi \rightarrow(\psi \rightarrow \chi), \gamma \rightarrow(\delta \rightarrow \chi) \quad \Rightarrow \quad \varphi \rightarrow(\gamma \rightarrow(\psi \vee \delta \rightarrow \chi))$

$$
\varphi \rightarrow \psi \Rightarrow \varphi \rightarrow \psi \vee \chi
$$$$
\varphi \rightarrow \psi \Rightarrow \varphi \rightarrow \chi \vee \psi
$$ 
Alternatively, instead of sequents 1.2.1. - 1.4.3., Suszko considers a set of 8 sequents which are also sufficient to capture the (classical) meaning of $\wedge, \vee, \leftrightarrow:$

$1.2 .1^{\prime}$.

$$
\begin{array}{lrl}
1.2 .1^{\prime} . & \varphi \rightarrow(\psi \rightarrow \chi) & \Rightarrow \varphi \wedge \psi \rightarrow \chi \\
1.2 .2^{\prime} . & \varphi \wedge \psi \rightarrow \chi & \Rightarrow \varphi \rightarrow(\psi \rightarrow \chi) \\
1.3 .1^{\prime} . & \varphi \rightarrow(\psi \rightarrow \chi), \varphi \rightarrow(\chi \rightarrow \psi) & \Rightarrow \varphi \rightarrow(\psi \leftrightarrow \chi) \\
1.3 .2^{\prime} . & \varphi \rightarrow(\psi \leftrightarrow \chi) & \Rightarrow \varphi \rightarrow(\psi \rightarrow \chi) \\
1.3 .3^{\prime} . & \varphi \rightarrow(\psi \leftrightarrow \chi) & \Rightarrow \varphi \rightarrow(\chi \rightarrow \psi) \\
1.4 .1^{\prime} . & \varphi \rightarrow \psi, \chi \rightarrow \psi & \Rightarrow \varphi \vee \chi \rightarrow \psi \\
1.4 .2^{\prime} . & \varphi \vee \psi \rightarrow \chi & \Rightarrow \varphi \rightarrow \chi \\
1.4 .3^{\prime} . & \varphi \vee \psi \rightarrow \chi & \Rightarrow \psi \rightarrow \chi
\end{array}
$$$$
1.4 .2^{\prime} \text {. }
$$$$
1.4 .3^{\prime} \text {. }
$$

The rules for quantifiers:

2.1. $\varphi[x / a] \Rightarrow \forall x \varphi$

2.2.1. $\forall x(\varphi \rightarrow \psi) \Rightarrow \varphi \rightarrow \forall x \psi$

2.2.2. $\forall x(\psi \rightarrow \varphi) \Rightarrow \exists x \psi \rightarrow \varphi$

2.3.1. $\varphi \rightarrow \forall x \psi \Rightarrow \varphi \rightarrow \psi[x / \tau]$

2.3.2. $\quad \exists x \varphi \rightarrow \psi \quad \Rightarrow \quad \varphi[x / \tau] \rightarrow \psi$

where in schemata 2.2.1. and 2.2.2 $\varphi$ has no occurence of $x$. Schema 2.1. shows that $\Rightarrow$ in Suszko's system does not correspond to the same relation of deducibility as in Gentzen's system.

Finally for identity we have two sequents (We omit a rule for definite descriptions; a full description of the system may be found in Indrzejczak [29]):

3.1. $\varphi \Rightarrow \exists x(x=a)$

3.2. $\varphi \Rightarrow \tau_{1}=\tau_{2} \rightarrow \varphi\left[\tau_{1} / / \tau_{2}\right]$

where $\tau_{i}$ represents any term and $\varphi$ in 3.1. is any formula. Also // in schema 3.2. means that any replacement is made not a substitution.

In order to derive other sequents from primitive ones Suszko uses several forms (actually 6) of the rule of substitution from Hilbert system but applied on the whole sequent, and 4 structural rules taken from Gentzen's LJ; i.e. weakening, permutation, contraction (in antecedents) and cut.

Suszko did not pay an attention to the form of representation of proof in his system. In what follows for compactness we display proofs in linear format since, in contrast to Gentzen's system, trees in Suszko's system tend to use quite long sequents already as leaves and it is hard to put them in the page.

It is an interesting feature of this system that only sequents with nonempty antecedent may be deduced from primitive sequents of the system by means of these structural rules, again, in contrast to Gentzen's system, where theses are sequents of the form $\Rightarrow \varphi$. Suszko's sequents naturally 
represent inference rules but in what way can we derive theses? After all his system is devised for classical logic not for purely inferential logics like e.g. Kleene's K3. Theses of classical logics are derivable as so called absolute sequents of the form $\varphi \Rightarrow \psi$, where $\varphi$ is an atomic formula not occuring as a subformula of $\psi$. Let us illustrate a proof of a thesis (i.e. an absolute sequent):

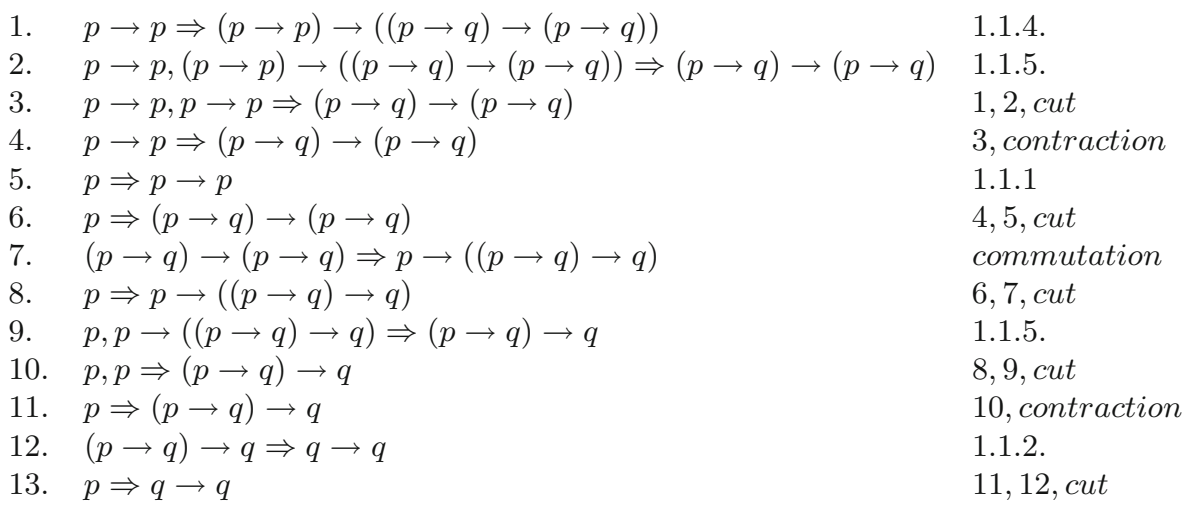

In line 7 . we have used derived sequent to slightly shorten the proof. Note, by the way, that a proof of $q \Rightarrow q$ is straightforward in Suszko's system (one cut on suitable instances of 1.1.1. and 1.1.5.), but we cannot apply Deduction Theorem to obtain an absolute sequent from resulting sequent.

In practice, Suszko's system treated as a kind of ordinary SC has a serious drawback concerning proof display. It may be partly overcome if we apply linear format but still proofs tend to be not only quite lengthy but also operate on often long sequents. It may be simplified a bit if we define a proof not as a linear sequence of sequents, but a linear sequence of formulae, where the first lines contain elements of the antecedent (of a proved sequent) and the last line is a succedent. Hence primitive sequents are not items in proof but rather descriptions of primitive inference rules on formulae, as in ordinary Natural Deduction on formulae. The proof of $p \Rightarrow q \rightarrow q$ displayed above may be rewritten in a slightly more compact and readable way:
1. $p$
2. $p \rightarrow p$ assumed antecedent
3. $(p \rightarrow p) \rightarrow((p \rightarrow q) \rightarrow(p \rightarrow q))$
1,1.1.1.
4. $(p \rightarrow q) \rightarrow(p \rightarrow q)$
$2,1.1 .4$.
5. $p \rightarrow((p \rightarrow q) \rightarrow q)$
$2,3,1.1 .5$.
6. $(p \rightarrow q) \rightarrow q$
4, commutation
7. $q \rightarrow q$
$1,5,1.1 .5$.
$6,1.1 .2$. 
In justification column we only refer to the number of primitive or a name of derivable sequent, and lines where its antecedents are displayed. The application of cut, contraction e.t.c. are implicit. This form of displaying proofs makes his system more menageable (shorter proofs made of shorter lines) and obtained system is more in natural deduction spirit. But it is not a natural deduction system; there are no subordinate proofs and no rules for discharging assumptions which is essential in this type of systems. It rather makes his system in some respects similar to systems of linear reasoning implicit in Herbrand's procedure.

By the end of [61] Suszko made some remarks concerning formalization of some nonclassical propositional logics.

1. A system comprising of only sequents 1.1.1. - 1.1.5. provides a formalization of positive propositional logic.

2. Addition of Peirce Law:

$$
(\varphi \rightarrow \psi) \rightarrow \varphi \Rightarrow \varphi
$$

yields implicational classical logic.

3. Replacement of 1.1.7. in propositional part (1.1.1. - 1.4.3.) by any of:

$$
\begin{aligned}
\neg \varphi \rightarrow \neg \psi & \Rightarrow \quad(\varphi \rightarrow \neg \psi) \rightarrow \neg \psi \\
\varphi \rightarrow \psi \quad & \Rightarrow \quad(\varphi \rightarrow \neg \psi) \rightarrow \neg \varphi
\end{aligned}
$$

provides a formalization of intuitionistic propositional logic.

4. Finally, if in this system we replace also 1.1.6. by:

$$
(\varphi \rightarrow \neg \psi) \rightarrow \chi \Rightarrow \neg \varphi \rightarrow \chi
$$

it yields a formalization of minimal propositional logic of Kolmogorov, Johansson.

Suszko himself was not satisfied with his solution because the project of total elimination of axioms in favor of proper inference rules was not fully realised. Namely sequent 3.1. is an absolute sequent expressing cryptoaxiomatic rule. Hence although it holds for his system that if a sentence is derivable from any sentence, then it is analytic (i.e. a thesis), then the converse does not hold, at least when identity is concerned. But Suszko was mistaken in this respect. One may use e.g. sequents corresponding to Kalish/Montague [33] rules for identity:

$$
\begin{aligned}
\forall x(x=\tau \rightarrow \varphi(x)) & \Rightarrow \varphi(\tau) \\
\varphi(\tau) & \Rightarrow \forall x(x=\tau \rightarrow \varphi(x))
\end{aligned}
$$


These sequents express proper rules in the sense of Suszko and are sufficient to obtain a complete system for first-order logic with identity. So in fact for Suszko's system with these rules replacing 3.1. and 3.2. it holds as well, that if a sentence is analytic, then it is derivable from any sentence.

\section{Mixed type}

Between the extrema of type one and two we can find a plethora of SC which use freely primitive sequents and rules to gain more flexibility in proof search. There is a great many possible combinations with some constants characterised only by means of rules and other only by means of sequents, or the same constants partly characterised by rules and partly by sequents. One can find considerations on these matters in Bernays [7], Popper [46] and many other logicians. Below we will illustrate the point with some examples.

In fact, the first solution of this kind is already in Gentzen [22], where he noticed that many rules of LK may be replaced with primitive sequents:

$$
\begin{aligned}
& \text { - } \Rightarrow \varphi, \neg \varphi \\
& \text { - } \neg, \varphi \Rightarrow \\
& \text { - } \varphi \wedge \psi \Rightarrow \varphi \\
& \text { - } \varphi \wedge \psi \Rightarrow \psi \\
& \text { - } \varphi, \psi \Rightarrow \varphi \wedge \psi \\
& \text { - } \varphi \Rightarrow \varphi \vee \psi \\
& \text { - } \psi \Rightarrow \varphi \vee \psi \\
& \text { - } \varphi \vee \psi \Rightarrow \varphi, \psi \\
& \text { - } \varphi \rightarrow \psi, \varphi \Rightarrow \psi \\
& \text { - } \forall x \varphi \Rightarrow \varphi[x / a] \\
& \text { - } \varphi[x / a] \Rightarrow x \varphi
\end{aligned}
$$

This list provides the basis for total elimination of rules for $\neg, \wedge, \vee$; there remain only three logical rules: $(\Rightarrow \rightarrow),(\Rightarrow \forall)$ and $(\exists \Rightarrow)$. Structural rules and axiomatic sequent remain with no changes. Let $\mathrm{LK}^{*}$ denotes the variant of LK, where all logical rules (except three abovementioned) were replaced with suitable primitive sequents. One can prove that:

$$
\vdash_{L K} \Gamma \Rightarrow \Delta \text { iff } \vdash_{L K^{*}} \Gamma \Rightarrow \Delta \text {. }
$$

Clearly Cut is not eliminable in $\mathrm{LK}^{*}$ since Modus Ponens is present only in weak form, i.e. as a sequent representing its inferential form, not in strong form, i.e. as a sequent rule (like in Gentzen's system described in section 2.1.). 


\subsection{Kleene and sequential representation of standard Natural Deduction}

In his well known textbook [35] (also [36]) Kleene introduces Hilbert system as basic but soon proves a lot of secondary rules of introduction and elimination which together provide adequate formalization of classical logic. In contrast to Gentzen's system and to most other described above, Kleene uses intuitonistic sequents but with antecedent as sets of formulae. $\wedge$ and $\rightarrow$ is characterised as in $\mathrm{LK}^{*}$, i.e. by means of 3 sequents for $\wedge$, and one sequent representing inferential version of Modus Ponens and one rule representing Deduction Theorem. For $\vee$ he also uses two sequents but elimination cannot be represented by sequent $\varphi \vee \psi \Rightarrow \psi, \chi$ (two formulae in succedent are not allowed) hence he uses a rule $(\vee \Rightarrow)$ with the same antecedents. For negation he applies two sequents: $\varphi, \neg \varphi \Rightarrow \psi, \neg \neg \varphi \Rightarrow \varphi$ and a rule $\Gamma, \varphi \Rightarrow \psi ; \Gamma, \varphi \Rightarrow \neg \psi / \Gamma \Rightarrow \neg \varphi$. For quantifiers he uses two sequents and two rules; exactly as in LK*.

It is easily provable that if we add to Kleene's set of sequents and rules three elements, namely: sequent $\varphi \Rightarrow \varphi$, rule of weakening in antecedent, and cut, we obtain adequate characterization of classical logic. Permutation and contraction are not needed since antecedents of sequents are sets. Cut is not eliminable, exactly for the same reason as in LK*

Such a system is very close to standard system NK of Natural Deduction as defined by Gentzen. The difference is that in the latter we operate (by means of inference rules) on formulae in the proof, whereas in Kleene's — if precisely formalised - we operate directly on sequents (representing inference rules) by means of sequent rules (representing prof construction rules of natural deduction). Hence sequents correspond to inference rules of standard natural deduction, and sequent rules correspond to proof construction rules of standard natural deduction. These correspondence is exact in case of $\wedge, \vee$ and $\rightarrow$ (with the only difference that in Kleene's system $(\vee \Rightarrow)$ has the same antecedents, whereas in Gentzen's natural deduction $(\vee E)$ is context-free). If we want to obtain a system where the correspondence is full we must make the following changes:

1. In case of negation we must use: $\varphi, \neg \varphi \Rightarrow \perp$ and $\perp \Rightarrow \varphi$ instead $\varphi, \neg \varphi \Rightarrow \psi$, and keep $\neg \neg \varphi \Rightarrow \varphi$ for classical logic. Instead of Kleene's rule we must use rule: $\Gamma, \varphi \Rightarrow \perp / \Gamma \Rightarrow \neg \varphi$. These changes follows from the fact that Kleene does not use $\perp$. One can also introduce a sequent rule corresponding to $(\neg E)$ which makes a sequent $\neg \neg \varphi \Rightarrow \varphi$ dispensable.

2. In case of quantifiers instead of $(\exists \Rightarrow)$ one can use the counterpart of $(\exists E$ ) of the form: $\Gamma \Rightarrow \exists x \varphi ; \Delta, \varphi[x / a] \Rightarrow \psi / \Gamma, \Delta \Rightarrow \psi$ with side condition 
to the effect that $a$ is not in $\varphi, \psi$ and $\Gamma, \Delta .(\forall D)$ is formulated as inference rule in Gentzen's natural deduction but with global side condition referring to active assumption, hence it is naturally represented as a sequent rule $(\Rightarrow \forall)$. Both sequents from $\mathrm{LK}^{*}$ correspond to $(\forall E)$ and $(\exists D)$.

This way we obtain another form of sequent Natural Deduction but significantly different from the Gentzen's system from section 2.1. Both systems operate on sequents (although in Suppes' variant this feature seems to be obscured) not on formulae like standard systems of Natural Deduction ${ }^{16}$. But in Gentzen's system from section 2.1. all constants are characterised by means of rules and, when compared with standard Natural Deduction, there is no difference between inference and proof construction rules. In the present system, constants are characterised either by sequents or by rules, and as a result we have a strict correspondence between sequents and inference rules of standard Natural Deduction on the one hand, and between sequent rules and proof construction rules of standard Natural Deduction.

\subsection{Hasenjaeger's system}

Hasenjaeger [24] contains SC defined on intuitionistic sequents with sequences in antecedents for classical propositional logic. Except structural rules on antecedents and Cut, only $\rightarrow$ is characterised by means of rules: $(\Rightarrow \rightarrow)$ and its converse instead of $(\rightarrow \Rightarrow)$; as we know (see subsection 2.5. on Došen's system) it yields adequate characterisation of intuitionistic implication, and after addition of suitable characterization of classical negation it suffices to obtain classical implication too.

Remaining connectives are characterised by means of primitive sequents: $\wedge$ as in $\mathrm{LK}^{*}$, similarly $\vee$ in succedent. A sequent characterising $\vee$ in antecedent must be different because only one formula in succedent is allowed. We have a sequent of the form:

$$
\varphi \rightarrow \chi, \psi \rightarrow \chi, \varphi \vee \psi \Rightarrow \chi
$$

For negation we have also different characterization:

- $\varphi, \neg \varphi \Rightarrow \psi$

- $\varphi \rightarrow \psi, \neg \varphi \rightarrow \psi \Rightarrow \psi$

The first sequent is the same as in $\mathrm{LK}^{*}$ but arbitrary $\psi$ is added since Hasenjaeger does not admit empty succedents. The second sequent replaces $\Rightarrow \varphi, \neg \varphi$ because more formulae are not admitted in succedents.

\footnotetext{
${ }^{16}$ I mean here NK of Gentzen [22] and the system of Jaśkowski.
} 
In this system the number of rules is reduced which makes it more similar to systems belonging to the second type. In fact, we can go further in this direction - we can introduce a sequent representing inferential Modus Ponens instead of the converse of $(\Rightarrow \rightarrow)$; such a solution is in the system of Surma [59] (but his selection of primitive sequents is slightly different).

It is easy to prove the adequacy of Hasenjaeger's system; it suffices to show its equivalence with LK*. In the latter one can easily deduce both sequents for $\neg$ and $\vee$ which replace Gentzen's sequents with two formulae in succedents. It gives a system strong enough to cover intuitionistic propositional logic (because of structural restriction on sequents). Hence it is sufficient to prove a law of excluded middle, here is the proof:



\subsection{Rieger's system}

The original SC was provided by Rieger [49]. He uses sequents which admit exactly one formula on both sides of a sequent. His set of primitive sequents comprises:

- $\varphi \Rightarrow \varphi$

- $\varphi \Rightarrow \varphi \vee \psi$

- $\varphi \Rightarrow \psi \vee \varphi$

- $\varphi \wedge \psi \Rightarrow \varphi$

- $\psi \wedge \varphi \Rightarrow \varphi$

- $\varphi \wedge(\psi \vee \chi) \Rightarrow(\varphi \wedge \psi) \vee(\varphi \wedge \chi)$

- $(\varphi \vee \psi) \wedge(\varphi \vee \chi) \Rightarrow \varphi \vee(\psi \wedge \chi)$

- $\neg \varphi \wedge \varphi \Rightarrow \psi$

- $\psi \Rightarrow \varphi \vee \neg \varphi$

- $\neg \varphi \vee \psi \Rightarrow \varphi \rightarrow \psi$

- $\varphi \rightarrow \psi \Rightarrow \neg \varphi \vee \psi$

- $\forall x \varphi \Rightarrow \varphi[x / a]$

- $\varphi[x / a] \Rightarrow \exists x \varphi$ 
Additionaly we have the following rules:

$$
\begin{aligned}
& (T R) \frac{\varphi \Rightarrow \psi \quad \psi \Rightarrow \chi}{\varphi \Rightarrow \chi} \\
& (\Rightarrow \wedge) \frac{\chi \Rightarrow \varphi \quad \chi \Rightarrow \psi}{\chi \Rightarrow \varphi \wedge \psi} \\
& (\vee \Rightarrow) \frac{\varphi \Rightarrow \chi \quad \psi \Rightarrow \chi}{\varphi \vee \psi \Rightarrow \chi} \\
& (\Rightarrow \forall)^{1} \quad \frac{\psi \Rightarrow \varphi}{\psi \Rightarrow \forall x \varphi} \\
& (\exists \Rightarrow)^{1} \quad \frac{\varphi \Rightarrow \psi}{\exists x \varphi \Rightarrow \psi}
\end{aligned}
$$

side conditions:

1. $x$ is not free in $\psi$.

Proof is defined as the sequence of such sequents. Similarly as in Suszko's system a proof of a sequent with empty antecedent is not possible. Since Rieger is interested in proofs of theorems in elementary theories he admits that $\varphi$ is a thesis of theory $\mathrm{T}$ iff a sequent $A \Rightarrow \varphi$ is provable, where $A$ is a conjunction of some instances of axioms from T. In contrast to Suszko's solution, in Rieger's system one can prove deduction theorem of the form: $\varphi \Rightarrow \psi$ iff $\chi \Rightarrow \varphi \rightarrow \psi$, where $\chi$ is arbitrary formula.

The decision that sequents of the system are restricted this way leads to some complications in proofs and in characterisation of constants in rules. For example, Rieger needs separate sequents to express some distribution laws (their converses are provable) for completeness. But this decision is neither arbitrary nor artificial from the standpoint of Rieger's purposes. His work is concerned mainly with algebraic aspects of classical logic and such sequents and rules more directly express these features. Rieger introduces also sequents of the form $\varphi \Leftrightarrow \psi$ for direct dealing with identities in algebraic structures. Similar solution is applied in Dunn and Hardegree [14], where $\mathrm{SC}$ for equivalential logics are considered.

\section{Conclusion}

We have focused on nonstandard systems designed for classical logic and only mentioned in passing some solutions for nonclassical logics. In fact, most of them belong rather to category of generalised sequent calculi but some of them, although developed in the context of mainstream study of standard SC (cut-elimination matters, e.t.c.), could be also classified as nonstandard. One may take as examples many formalizations of modal logic like GL or S4.3. where rules proposed for introduction of $\square$ in the succedent are far from being standard in the sense of Gentzen. 
The results reported in this paper were realised within a project financed by the National Science Centre, Poland (decision no. DEC-2011/03/B/HS1/ 04366).

\section{References}

[1] Ajdukiewicz, K., Sprache und Sinn, Erkenntniss IV:100-138, 1934.

[2] Anderson, A.R., and N.D. Belnap, Entailment: the Logic of Relewance and Necessity, vol. I, Princeton University Press, Princeton 1975.

[3] Andrews, P. B., An Introduction to Mathematical Logic and Type Theory: to Truth through Proof, Harcourt Academic Press, Orlando 1986.

[4] Avron, A., Simple Consequence Relations, Information and Computation 92:105$139,1991$.

[5] Avron, A., The Method of Hypersequents in the Proof Theory of Propositional NonClassical Logics, in W. Hodges et al. (eds.), Logic: From Foundations to Applications, Oxford Science Publication, Oxford, 1996, pp. 1-32.

[6] Belnap, N. D., Display Logic, Journal of Philosophical Logic 11:375-417, 1982.

[7] Bernays, P., Betrachtungen zum Sequenzen-Kalkul, in A. T. Tymieniecka (ed.), Contributions to Logic and Methodology in honor of J. M. Bocheński, North-Holland, Amsterdam 1965, pp. 1-44.

[8] Blamey, S., and L. Humberstone, A Perspective on Modal Sequent Logic, Publications of the Research Institute for Mathematical Sciences, Kyoto University 27:763$782,1991$.

[9] Carnielli, W. A., On Sequents and Tableaux for Many-valued Logics, Journal of Non-Classical Logic 8(1):59-76, 1991.

[10] Ciabattoni, A., R. Ramanayake, and H. Wansing, Hypersequent and Display Calculi - a unified pespective, Studia Logica, this issue.

[11] Curry, H. B., Foundations of Mathematical Logic, McGraw-Hill, New York 1963.

[12] Došen, K., Sequent-systems for Modal Logic, Journal of Symbolic Logic 50:149-159, 1985.

[13] Dos̆en, K., Logical constants as punctuation marks, Notre Dame Journal of Formal Logic 30:362-381, 1989.

[14] Dunn, J.M., and G.M. Hardegree, Algebraic Methods in Philosophical Logic, Clarendon, Oxford 2001.

[15] Ebbinghaus, H. D., J. Flum, and W. Thomas, Mathematical Logic, Springer, Berlin 1984.

[16] Ershow, Y.L., and E. A. Palyutin, Mathematical Logic, MIR, Moscow 1984.

[17] Feys, R., and J. LAdRIERE, Supplementary notes, in Recherches sur la deduction logique, french translation of Gentzen, Press Univ. de France, Paris 1955.

[18] Forbes, G., Modern Logic, New York 2001.

[19] Gabbay, D., LDS - Labelled Deductive Systems, Clarendon Press, Oxford 1996.

[20] Garson, J.W. Modal Logic for Philosophers, Cambridge University Press, Cambridge 2006.

[21] Gentzen, G., Über die Existenz unabhängiger Axiomensysteme zu unendlichen Satzsystemen, Mathematische Annalen 107:329-350, 1932. 
[22] Gentzen, G., Untersuchungen über das Logische Schliessen, Mathematische Zeitschrift 39:176-210 and 39:405-431, 1934.

[23] Gentzen, G., Die Widerspruchsfreiheit der reinen Zahlentheorie, Mathematische Annalen 112:493-565, 1936.

[24] Hasenjaeger, G., Introduction to the Basic Concepts and Problems of Modern Logic, Reidel, Dordrecht 1972.

[25] Hermes, H., Einführung in die Mathematische Logik, Teubner, Stuttgart 1963.

[26] Hertz, P., Über Axiomensysteme für beliebige Satzsysteme, Mathematische Annalen 101:457-514, 1929.

[27] Hudelmayer, J., and P. Schroeder-Heister, Classical Lambek Calculus, in P. Baumgartner, R. Hähnle, and J. Possega (eds.), Theorem Proving with Analytic Tableaux and Related Methods. 4th International Workshop, TABLEAUX'95 (St. Goar, May 7-10, 1995), Springer LNAI, Bd. 918, 1995, pp. 247-262.

[28] Indrzejczak, A., Generalised Sequent Calculus for Propositional Modal Logics, Logica Trianguli 1:15-31, 1997.

[29] Indrzejczak, A., Suszko's Contribution to the Theory of Nonaxiomatic Proof Systems, Bulletin of the Section of logic 38(3-4):151-162, 2009.

[30] Indrzejczak, A., Natural Deduction, Hybrid Systems and Modal Logics, Springer 2010 .

[31] Indrzejczak, A., Rachunki sekwentowe w logice klasycznej, Wyd. UŁ, Łódź 2013.

[32] JAŚKowski, S., On the Rules of Suppositions in Formal Logic Studia Logica 1:5-32, 1934.

[33] Kalish, D., and R. Montague, Logic, Techniques of Formal Reasoning, Harcourt, Brace and World, New York 1964.

[34] Kashima, R., Cut-free sequent calculi for some tense logics, Studia Logica 53:119-135, 1994.

[35] Kleene, S. C., Introduction to Metamathematics, North Holland, Amsterdam 1952.

[36] Kleene, S. C., Mathematical Logic, Willey, New York 1967.

[37] Leblanc, H., Proof routines for the propositional calculus, Notre Dame Journal of Formal Logic 4(2):81-104, 1963.

[38] Leblanc, H., Two separation theorems for natural deduction, Notre Dame Journal of Formal Logic 7(2):81-104, 1966.

[39] Lemmon, E. J., Beginning Logic, Nelson, London 1965.

[40] Leszczyńska-Jasion, D., M. Urbański, and A. Wiśniewski, Socratic Trees, Studia Logica 101(5):959-986, 2013.

[41] ŁaWrow, I. A., and L. L. Maksimowa, Zadania z teorii mnogości, logiki matematycznej i teorii algorytmów, PWN, Warszawa 2004.

[42] Negri, S., and J. von Plato, Structural Proof Theory, Cambridge University Press, Cambridge 2001.

[43] Nishimura, H., A Study of Some Tense Logics by Gentzen's Sequential Method, Publications of the Research Institute for Mathematical Sciences, Kyoto University 16:343-353, 1980.

[44] von Plato, J., Natural deduction with general elimination rules, Archiv for Mathematical Logic 40:541-567.

[45] Poggiolesi, F., Gentzen Calculi for Modal Propositional Logic, Springer 2011. 
[46] Popper, K., Logic without assumptions, Proceedings of the Aristotelian Society 47:251-292, 1947.

[47] Popper, K., New foundations for Logic, Mind 56:1947.

[48] Quine, W. Van O., Methods of Logic, Colt, New York 1950.

[49] Rieger, L., Algebraic Methods of Mathematical Logic, Academia, Prague 1967.

[50] Rousseau, G., Sequents in Many Valued Logic, Fundamenta Mathematicae LX(1):22-23, 1967.

[51] Schroeder-Heister, P., Popper's theory of deductive inference and the concept of a logical constant, History and Philosophy of Logic 5:79-110, 1984.

[52] Schroeder-Heister, P., Resolution and the origins of structural reasoning: early proof-theoretic ideas of Hertz and Gentzen, The Bulletin of Symbolic Logic 8(2):246$265,2002$.

[53] Schroeder-Heister, P., Popper's structuralist theory of logic, in I. Jarvie, K. Milford, D. Miller (eds.), Karl Popper: A Centenary Assesment, vol III: Science, Ashgate Publishing: Aldershot 2006, pp. 17-36.

[54] Schütte, K., Proof Theory, Springer, Berlin 1977.

[55] Sсотт, D., Rules and derived rules, in S. Stenlund (ed.), Logical Theory and Semantical Analysis, 1974, pp. 147-161.

[56] Smullyan, R., First-Order Logic, Springer 1968.

[57] Stouppa P., A deep inference system for the modal logic S5, Studia Logica 85:199$214,2007$.

[58] Suppes P., Introduction to Logic, Van Nostrand, Princeton 1957.

[59] Surma, S. J., Wprowadzenie do metamatematyki T. I, Kraków 1965.

[60] Suszko, R., W sprawie logiki bez aksjomatów, Kwartalnik Filozoficzny 17(3/4):199205, 1948.

[61] Suszko, R., O analitycznych aksjomatach $i$ logicznych regutach wnioskowania, Poznańskie Towarzystwo Przyjaciół Nauk, Prace Komisji Filozoficznej, 7/5, 1949.

[62] Suszko, R., Formalna teoria wartości logicznych, Studia Logica 6:145-320, 1957.

[63] Tait, W. W., Normal Derivability in Classical Logic, in The Sintax and Semantics of Infinitary Languages, LNM 72, 1968, pp. 204-236.

[64] Wansing, H., Displaying Modal Logics, Kluwer Academic Publishers, Dordrecht 1999.

[65] Wansing, H., Sequent Systems for Modal Logics, in D. Gabbay, F. Guenthner (eds.), Handbook of Philosophical Logic, vol IV, Reidel Publishing Company, Dordrecht 2002, pp. 89-133.

[66] Wiśniewski, A., Socratic Proofs, Journal of Philosophical Logic 33(3):299-326, 2004.

ANDRZEJ INDRZEJCZAK

Department of Logic

University of Łódź

Kopcinskiego 16/18

90-232 Łódź, Poland

indrzej@filozof.uni.lodz.pl

Open Access This article is distributed under the terms of the Creative Commons Attribution License which permits any use, distribution, and reproduction in any medium, provided the original author(s) and the source are credited. 